\title{
Une expérience en matière de formation en radioprotection
}

\author{
J. FLUCHERE* \\ (Manuscrit reçu le 13 juin 1983)
}

\begin{abstract}
RÉSUMÉ
L'auteur, ancien directeur du centre de formation d'EdF du Bugey, nous fait part de ses réflexions sur la formation du personnel DATR dont l'effectif a quadruplé en 6 ans et dont la qualification initiale est extrêmement variable.

II indique les choix qui ont été faits et les actions concrètes sur lesquelles, l'expérience l'a montré, il était important d'insister. II attire aussi l'attention sur les problèmes psychologiques dont il a fallu tenir compte pour travailler de façon efficace.
\end{abstract}

\section{ABSTRACT}

The author, a former head of an EdF training center, gives his thoughts on the training of EdF nuclear power plant radiation workers whose number was increased fourfold in 6 years and whose initial qualifications varied considerably.

The orientation already taken and the directions that experience has suggested to be promising are indicated. Attention is also drawn on psychological problems that should be taken into consideration in order to work efficiently.

La population des agents EdF, travailleurs "directement affectés aux travaux sous rayonnements ionisants" (DATR), est passée de 1700 en 1976 à 7000 en 1982. Un tel accroissement impose, bien sûr, le développement des méthodes et moyens de formation, mais, au-delà des chiffres, la formation en radioprotection, comme toute action de formation à la sécurité dans le travail, pose un problème pédagogique délicat dont les trois composantes : objectif, population, difficultés, doivent être soigneusement examinés avant de dégager les solutions.

\section{OBJECTIF : RÉDUIRE LES DOSES}

L'objectif visé consiste à dispenser une formation qui permette de réaliser les activités quotidiennes en zone contrôlée en réduisant au maximum les doses reçues par les intervenants et en restant toujours audessous de la "limite de dose".

* Electricité de France, Centre de production nucléaire du Bugey. 
Ainsi défini, l'objectif est très vaste. On peut, cependant, le préciser en retenant que la plupart des tâches à prendre en compte sont liées à l'exploitation et à l'entretien de matériels constituant des circuits de fluides analogues à ceux déjà en service dans les centrales conventionnelles. II s'agit de faire en sorte qu'aux gestes professionnels habituels s'ajoutent les attitudes et les actions indispensables pour se protéger des rayonnements ionisants.

Le formateur doit donc posséder une bonne connaissance des installations et dresser une liste des activités caractérisées par nature de risques, par exemple :

- travaux dans les locaux où le débit de dose est élevé ou peut varier brusquement ;

- ouverture de capacités ayant contenu des fluides ou des gaz radioactifs ;

- interventions sur des matériels contaminés en surface ;

- travaux à l'intérieur de locaux ou de capacités dans lesquels peut régner une contamination atmosphérique.

L'établissement de cette liste est indispensable à la préparation d'un enseignement adapté aux problèmes rencontrés.

\section{LA POPULATION INTÉRESSÉE ? TRĖS HÉTÉROGÉNE}

On y trouve :

- les agents de conduite qui surveillent et exploitent les installations ;

- les agents d'entretien qui interviennent dans les spécialités de mécanique, électricité, chaudronnerie et services généraux ;

- les agents des services techniques qui réalisent des essais, font des analyses chimiques ou radiochimiques et manutentionnent les combustibles.

A cette variété dans les activités professionnelles s'ajoute un large éventail des niveaux de qualification qui vont de l'agent d'exécution à l'ingénieur.

Cette énumération impose au pédagogue de développer une formation qui puisse être accessible à tous. Les connaissances théoriques utilisées ne doivent donc pas dépasser celles exigées pour l'obtention du CAP. Après expérience, cette exigence a probablement été bénéfique en privant les instructeurs du refuge pernicieux que constituent les grands développements théoriques.

\section{PRINCIPALES DIFFICULTÉS : L'IMPERCEPTIBILITÉ ET LA CRAINTE}

La caractéristique essentielle est qu'il s'agit d'une formation à la sécurité, c'est-à-dire que les acteurs vont ensuite être impliqués physiquement. Rien à voir avec un cours de chimie ou de mécanique, la formation en radioprotection comme la formation aux travaux sous tension ou aux travaux en hauteur est destinée à des agents qui vont être placés 
face à un risque et qui vont devoir le surmonter sans (ou avec un minimum) de dommage.

Les difficultés sont essentiellement liées au fait que le risque est nouveau et que les rayonnements sont imperceptibles à nos sens. Les hommes n'analysent jamais de façon rationnelle un nouveau danger. La réaction instinctive est la peur et elle ne conduit pas toujours aux attitudes et gestes pertinents. Certains attribuent ce comportement au conflit permanent qui oppose les deux cerveaux de l'homme: le néocortex, siège de la rationalité acquise, et le cortex ancien véhiculant l'inné et transmettant nos instincts. La raison ne peut l'emporter sur le comportement instinctif qu'après formation et entraînement. En radioprotection, la difficulté est d'autant plus grande que le risque perçu est totalement imaginé par le sujet. L'individu qui est chargé d'un travail en hauteur appréhende de façon directe le risque de chute, l'homme a naturellement peur du vide et les chutes diverses qu'il a subies constituent une expérience personelle des conséquences de ce risque.

Dans le domaine des rayonnements ionisants, il en va tout autrement. N'étant pas directement perceptibles, la crainte qu'ils engendrent est relativement récente. Elle résulte tout aussi probablement des méfaits réels constatés sur les pionniers que du tapage entretenu par les médias. Et dans ce domaine, la clarté n'a pas été la règle. Les débats compliqués et contradictoires, les déclarations divergentes des scientifiques sur les risques somatiques et génétiques, les querelles d'école sur la notion de seuil, de proportionalité entre la dose et l'effet, de cumul des doses n'ont fait qu'accroître la confusion dans l'esprit du public. A cela s'ajoute P'influence du mouvement antinucléaire qui a érigé ce risque en mythe social et en a fait son principal argument de lutte. Et dans ce combat, les propagandistes n'ont pas fait dans la nuance : tout le monde a pu voir ces tracts représentants des hommes et des femmes amputés, des enfants atteints de malformations et même le globe terrestre réduit à l'état de désert. Sûrement pas du meilleur goût, cette imagerie a profondément marqué les esprits, entraînant un très grand décalage entre le risque réel et le risque perçu. Symboles d'une vie "dénaturée" au même titre que les colorants alimentaires, le public accepte moins bien ces risques que ceux autrement plus importants pris quotidiennement en utilisant les moyens de transports sans parler de l'usage de l'alcool et du tabac.

Enfin, il faut noter qu'au risque d'agression externe par irradiation s'ajoute celui de contamination interne mal vécu psychologiquement. Le pourquoi est probablement difficile à bien connaître mais le fait est patent et doit être pris en considération.

Cet examen une fois terminé, le formateur peut alors édifier sa stratégie éducative en privilégiant trois grands axes :

- la démythification du risque

- la perception des rayonnements

- l'entraînement par mise en situation réelle de travail. 


\section{La démythification du risque}

II est superflu d'ajouter un discours confus et compliqué à tous ceux que les stagiaires ont déjà pu lire et entendre. Le principe consiste plutôt à utiliser la première séance du stage à faire parler les stagiaires sur leur représentation des rayonnements ionisants et des risques associés. II faut que la discussion soit ouverte, que le formateur soit à l'écoute, qu'il note bien les craintes, les angoisses, l'anxiété profonde, qu'il dégage les attentes des stagiaires et du groupe, qu'il fasse apparaître l'irrationalité de certains échanges mais sans porter de jugement de valeur et qu'il propose le plan du stage.

Cette première séance est primordiale. Elle permet d'établir un climat de confiance entre les stagiaires et les instructeurs, climat indispensable à l'expression spontanée des craintes, des doutes et des angoisses. Tout le stage doit baigner dans cette ambiance. Si l'on veut atteindre l'objectif de démythification, il faut que chacun puisse exprimer constamment ses problèmes et trouver toujours un instructeur attentif et compréhensif. Progressivement, l'atmosphère se détend, les problèmes sont abordés plus rationnellement, les débats perdent de leur passion pour gagner en clarté et le chantier école qui clôture le stage concrétise ce changement global d'attitude.

\section{La perception des rayonnements : passer de l'invisible au visible}

Faire percevoir un danger non perceptible à nos sens est finalement un cas courant en matière de sécurité. Les dangers présentés par l'électricité et certains gaz sont également imperceptibles aux sens humains et les méthodes de formation tout comme les techniques de prévention ont permis de surmonter cette difficulté. Comment? Tout simplement en faisant passer l'invisible dans le domaine perceptible par l'usage d'appareils simples à lecture facilement interprétable. En électricité, le voltmètre est l'exemple typique. Peu importe la définition scientifique du volt, il suffit de connaître les effets comparés entre une différence de potentiel de $48 \mathrm{~V}$ et une de $380 \mathrm{~V}$.

II faut qu'à force d'expériences les stagiaires s'habituent à la manipulation des appareils, aux unités de mesure et aux echelles employées. Alors, progressivement, se bâtit dans leur esprit une représentation mentale pertinente du risque. Tout se passe comme si l'appareil de mesure avait élargi leur champ de perception.

Le formateur doit s'entourer de l'appareillage courant et procéder expérimentalement. II faut privilégier la manipulation des appareils de détection. Les stagiaires doivent s'habituer à leur utilisation et à l'interprétation des valeurs lues. II faut également ne pas multiplier les types d'appareils (et c'est vrai dans toute action de sécurité) sinon le risque est grand de rendre plus difficile la compréhension, de décourager les stagiaires ou de conduire à de fausses acquisitions. A titre d'exemple, on peut citer les difficultés rencontrées avec les appareils de mesure de contamination gradués en coups par seconde. Les agents sont rebutés par leur usage, car l'interprétation des mesures est toujours délicate et demande une grande habitude. 
L'étape de la perception étant passée, les stagiaires sont à même d'aborder les principes de protection. Comme précédemment, l'approche de la compréhension de la protection contre l'irradiation par la distance et Pinterposition d'écrans est faite d'une manière expérimentale. Les stagiaires doivent manipuler écrans et appareils pour assimiler ces notions simples. Toutes les démonstrations mathématiques, surtout satisfaisantes pour celui qui les fait, doivent être soigneusement releguées parce qu'elles sont incompréhensibles à la plupart, et qu'elles n'apportent donc rien à la démonstration et surtout parce que la réalité est presque toujours différente du modèle mathématique; par exemple, la source ponctuelle, représentation si simple et si commode pour les calculs, n'a pas de réalité physique en centrale nucléaire. II suffit que les stagiaires aient acquis définitivement la notion de protection par éloignement ou par écrans de densité différente.

La notion de protection contre les risques de contamination est toujours plus délicate. II faut d'abord procéder à l'inventaire des différents cas depuis la contamination atmosphérique par gaz ou par aérosols jusqu'à la contamination surfacique fixée et non fixée. Les techniques de décontamination et les moyens de protection étant différents suivant les cas, il est important que cet inventaire soit clair et toujours présent à l'esprit des participants. Il faut ensuite passer à la notion de risque lié à la contamination. Là se situe la difficulté. En effet, la tentation est toujours grande pour le formateur de se lancer dans des démonstrations sur la CMA des radionucléides, les périodes biologiques et radioactives, les organes critiques, les sondes de mesures et toutes autres considérations. Autant dire que, pour rigoureuse que soit cette approche, elle risque de déclencher la perplexité générale, et surtout de ne pas apporter beaucoup à la formation en radioprotection des agents.

On doit se contenter de donner une bonne représentation mentale de la $L_{M A^{(1)}}$, s'assurer de la connaissance des gestes de décontamination, de sa nécessité selon les cas, et surtout de faire acquérir des critères stricts de choix des tenues individuelles, de la façon de les mettre, de les porter et de les enlever. II est plus important pour les travailleurs DATR de savoir enfiler une tenue complémentaire, respirer avec un appareil autonome, et se dévêtir correctement ensuite que de connaître la CMA du strontium 90 et l'organe critique.

\section{La mise en situation réelle de travail}

Le chantier école constitue l'étape la plus importante de cette formation. II représente non seulement l'application pratique des phases précédentes mais surtout l'entraînement indispensable par mise en situation réelle de travail.

Dans toute formation destinée à des individus qui, face à un risque, doivent surmonter leur appréhension, il faut passer par une phase d'entraînement. Quand on veut préparer des parachutistes, il faut les faire sauter. C'est le seul moyen pour les agents d'appréhender la réalité de la situation, d'acquérir le geste et le savoir-faire, de se rendre compte des erreurs et d'observer leur propre comportement.

(1) LMA : limite maximale admissible. 
Le chantier doit, si possible, se trouver en zone contrôlée et simuler une opération habituelle. II faut que les stagiaires s'habillent, préparent le chantier, réalisent le travail, procèdent aux travaux de repli et reviennent hors zone.

Les instructeurs doivent être très vigilants, observer les écarts et n'intervenir qu'au tout dernier moment. II n'est pas bon de trop guider les stagiaires qui doivent se prendre eux-mêmes en charge. En effet, la prise de conscience des défauts de leur comportement et des conséquences qu'ils peuvent entraîner est essentielle.

II faut aussi que les gestes de radioprotection deviennent, à force de répétition, des gestes habituels. L'action sûre et précise, adaptée au travail ainsi qu'au risque qui l'accompagne, inhibe l'appréhension et permet aux agents de travailler avec une vigilance intacte.

\section{L'ADAPTATION AUX NIVEAUX DE RESPONSABILITÉ}

La démarche pédagogique décrite dans ces trois volets est appliquée dans deux types de stages qui se complètent. Le premier stage appelé "Radioprotection 1 $1^{\text {er }}$ palier" est un stage destiné à tous les agents d'exécution. Tous les travailleurs DATR le suivent obligatoirement. Le deuxième stage, délivré en complément au premier, s'adresse à tous les chefs de travaux, c'est-à-dire aux responsables de l'exécution et du déroulement des chantiers. Chacun de ces stages dure une semaine et se déroule directement sur les sites nucléaires.

\section{FORMATEURS : DES HOMMES DE TERRAIN}

Les formateurs sont, la plupart du temps, des techniciens et contremaîtres des sections "sécurité-radioprotection" qui reçoivent à cette fin une formation pédagogique. La formation fait partie de leur activité professionnelle normale et, régulièrement, ils animent les sessions de sécurité et de radioprotection. Ce choix est délibéré, car il est fondamental que la formation soit irriguée par le vécu professionnel. II y va de la qualité de la formation, de son dépouillement de tout superflu et de la crédibilité des formateurs. D'autre part, il est important que le formateur constate sur le terrain les effets de ses actions de formation, rencontre les agents qu'il a entraîné et nourrisse son travail de technicien de la réflexion acquise pendant son travail d'animateur.

\section{CONCLUSION}

En conclusion, l'expérience montre que l'application pratique d'une technique ou d'un savoir-faire comme la radioprotection peut passer dans la vie quotidienne d'une grande population d'agents et cesser d'être 
considérée comme une science à part. Le développement d'une formation adaptée à la population concernée et aux activités déployées dans les centrales nucléaires, l'intégration de cette formation dans le "paquetage" traditionnel des actions de sécurité ont permis de franchir le cap des premières années d'exploitation d'un parc nucléaire important sans que l'on ait à déplorer d'accidents graves.

En matière de sécurité, le cas est probablement exceptionnel. 